\title{
Comparison of one-stage direct-to-implant with acellular dermal matrix and two-stage immediate implant-based breast reconstruction - a cohort study
}

\author{
Mette Eline Brunbjerg ${ }^{1,2} \wedge$, Thomas Bo Jensen ${ }^{1}$, Jens Overgaard $^{3} \wedge$, Peer Christiansen $^{1} \wedge$, \\ Tine Engberg Damsgaard ${ }^{2} \wedge$
}

${ }^{1}$ Department of Plastic and Breast Surgery, Aarhus University Hospital, Aarhus, Denmark; ${ }^{2}$ Department of Clinical Medicine, Aarhus University, Aarhus, Denmark; ${ }^{3}$ Department of Experimental Clinical Oncology, Aarhus University Hospital, Aarhus, Denmark

Contributions: (I) Conception and design: ME Brunbjerg, TE Damsgaard, P Christiansen; (II) Administrative support: TE Damsgaard, J Overgaard; (III) Provision of study materials or patients: TE Damsgaard, TB Jensen; (IV) Collection and assembly of data: ME Brunbjerg; (V) Data analysis and interpretation: ME Brunbjerg, TE Damsgaard; (VI) Manuscript writing: All authors; (VII) Final approval of manuscript: All authors.

Correspondence to: Mette Eline Brunbjerg, MD. Department of Plastic and Breast Surgery, Aarhus University Hospital, Palle Juul-Jensens Boulevard 99, Entrance C, CM419, DK - 8200 Aarhus N., Denmark. Email: metteeline.brunbjerg@gmail.com.

\begin{abstract}
Background: The use of acellular dermal matrix (ADM) in one-stage immediate implant-based breast reconstruction (BR) may offer advantages over the two-stage expander-to-implant technique, but literature shows conflicting results. The aim of the present study was to compare these two techniques for immediate implant-based BR regarding postoperative complications, aesthetic correction procedures and aesthetic outcome.

Methods: The study was designed as an observational cohort study with 44 participants admitted for immediate implant-based BR at Department of Plastic Surgery, Aarhus University Hospital, Denmark. 21 patients underwent BR with a one-stage direct-to-implant technique using ADM and 23 patients underwent BR with a two-stage expander-to-implant technique. Follow-up time was 2 years.
\end{abstract}

Results: The risk of implant loss was equal between groups; one-stage group 16\% and two-stage group $17 \%$ whereas the risk of implant exchange (but not loss of BR) was $13 \%$ in the one-stage group compared to $7 \%$ in the two-stage group. The risk of at least one major complication were equal between groups; $28 \%$ and $24 \%$ but the risk of at least one minor complication was significantly higher in the two-stage group (41\%) compared to the one-stage group (3\%). Number of aesthetic corrections were equally frequent in the two treatment groups (one-stage group 1.8, two-stage group 1.5). Patient and investigator assessed aesthetic outcome was very high in both groups as well as the degree of symmetry between breasts. No capsular contracture Baker grade 3 or 4 was observed.

Conclusions: The present study design sets limitations for drawing wide conclusions. This study did not reveal any significant differences between the two breast reconstructive techniques besides a higher risk of minor complications in the two-stage group, that did, however, not lead to a higher risk of implant loss. With equally high satisfaction with the aesthetic result and no significant difference in number of aesthetic corrections between the two groups we suggest, that the one-stage approach using ADM may be feasible and allows the patient to achieve an implant-based BR with a minimum of surgeries and outpatient visits. The study was registered in ClinicalTrials.gov (NCT04209010).

Keywords: Implant-based breast reconstruction (BR); acellular dermal matrix (ADM); complications; aesthetic outcome

^ ORCID: Mette Eline Brunbjerg, 0000-0003-1763-7617; Jens Overgaard, 0000-0002-0814-8179; Peer Christiansen, 0000-0003-27489240; Tine Engberg Damsgaard, 0000-0002-1627-9566. 
Submitted Jun 25, 2020. Accepted for publication Sep 30, 2020.

doi: $10.21037 /$ gs-20-581

View this article at: http://dx.doi.org/10.21037/gs-20-581

\section{Introduction}

Skin-sparing mastectomy provides optimal conditions for immediate reconstruction of an aesthetically satisfying breast. Even though autologous breast reconstruction (BR) has become very popular, some patients prefer less extensive surgery or are not candidates due to lack of tissue, comorbidity or worries about donorsite morbidity. For those patients, an implant-based BR may be the obvious choice. The use of acellular dermal matrix (ADM) to obtain onestage BR has revolutionized the concept of immediate implant-based BR after skin-sparing mastectomy (1). Attention has been drawn to advantages as improved control of inframammary fold position (2) and better lower pole projection (3) in comparison with the traditional two-stage expander to implant technique which can be associated with difficulties to achieve lower pole projection and ptosis (4). Furthermore, the two-stage method entails an extended course of treatment including multiple expansions and additional surgery for implant exchange. Despite more than two decades of research evaluating complications after implant-based BR with ADM the results are contradictory and level of evidence often low. It is often unclear which complications have been assessed and how they have been diagnosed, and also how and when capsular contracture and aesthetic outcome have been evaluated (5). Patients' perception of cosmetic outcome is a critical endpoint and the result of the BR should most likely be worth the effort and the struggles the women undergo in the BR treatment trajectory. Patients experiencing surgical complications after $\mathrm{BR}$ report suboptimal aesthetic outcome. This is reported to be more pronounced in patients undergoing implant-based BR compared to autologous reconstruction and BR after prophylactic mastectomy (6). This emphasizes the need for research regarding ways to decrease the complication rate and thereby improving the satisfaction with the BR.

The aim of the present study is to compare immediate implant-based BR using the one-stage technique with $\mathrm{ADM}$ with the two-stage expander to implant technique regarding postoperative complications, aesthetic correction procedures and aesthetic outcome.

We present the following article in accordance with the STROBE reporting checklist (available at http://dx.doi. org/10.21037/gs-20-581).

\section{Methods}

\section{Study design and participants}

The present study was designed as an observational cohort study with 44 participants. Eligible patients were all women admitted for immediate, implant-based BR following skin-sparing or nipple-sparing mastectomy and nipplesparing at the Department of Plastic and Breast Surgery, Aarhus University Hospital, Denmark over a period of 40 months. Patients were diagnosed with either breast cancer, ductal carcinoma in situ (DCIS) or were considered high risk for developing breast cancer. Inclusion criteria were mastectomy weight $\leq 600 \mathrm{~g}$, patient older than 18 years, tobacco abstinence $>4$ weeks prior to surgery (7), ability to complete the study questionnaire and for the twostage group; time to achieve two year follow-up visit after BR. Allocation to either one-stage BR or two-stage BR were based on enrolment time. Patients undergoing BR before December 2012 were assigned to two-stage BR and patients undergoing BR after December 2012 were assigned to onestage BR.

Follow-up time was 24 months. If explantation occurred the breast (in case of patient with bilateral BR and one explantation) or the patient was terminated and lost to follow-up. Participants underwent clinical examination and completed a study-specific questionnaire regarding aesthetic satisfaction with the result. Furthermore, a systematic review of patient records was performed to obtain information regarding complications.

All participants gave written informed consent. The trial was conducted in accordance with the Declaration of Helsinki (as revised in 2013). The Ethics Committee of the Central Region of Denmark (1-10-72-572-12) approved this study and it was submitted in ClinicalTrials.gov (NCT04209010).

\section{Recruitment}

As the one-stage approach was implemented as a standard care for immediate implant-based BR following skin- 


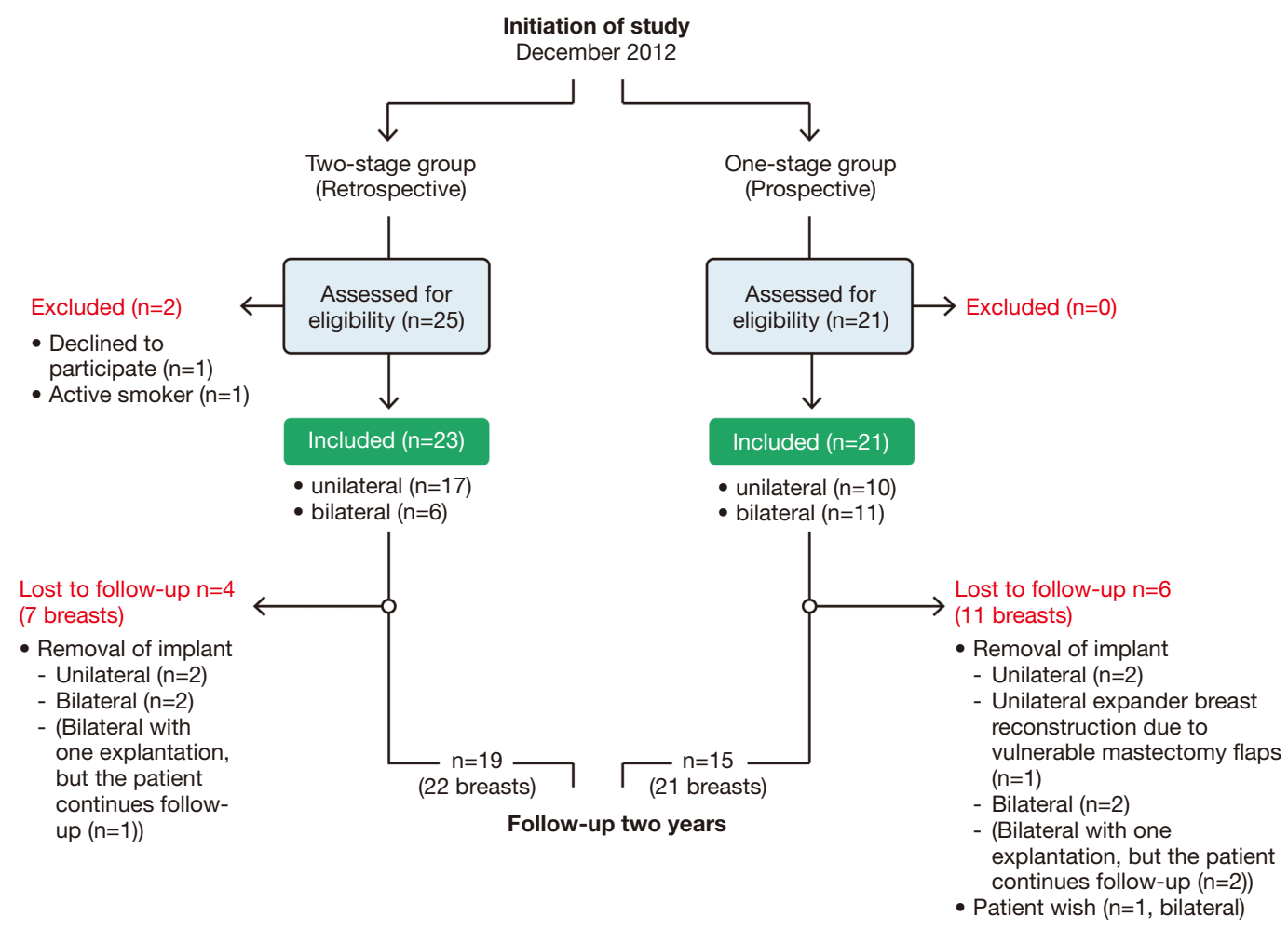

Figure 1 Flow diagram of the study participants.

sparing mastectomy, in December 2012, all eligible patients were offered participation in the one-stage group and inclusion continued consecutively until 21 patients were included. The two-stage cohort was established retrospectively. Patients that had undergone immediate implant-based BR following skin-sparing mastectomy with the two-stage expander to implant technique were identified using diagnosis- and procedure-related codes, records were examined and patients that fulfilled the inclusion criteria were identified and consecutively offered participation in the two-stage group. Inclusion continued retrospectively until 23 patients were included (Figure 1).

\section{Surgical techniques}

In all cases the implant or expander was placed behind the pectoralis muscle. The expanders used were Natrelle ${ }^{\circledR}$ with self-sealing dome port. The anatomical textured silicone gel-filled implants used were Eurosilicone ${ }^{\circledR}$ or Natrelle ${ }^{\circledR}$.

Surgical technique for one-stage immediate BR using porcine ADM (Strattice ${ }^{\mathrm{TM}}$, LifeCell Corporation, Branchburg, NJ, USA) and fixed size silicone implant:
The pectoralis major muscle was elevated and divided inferomedial. A sizer was used to determine implant size considering the viability of mastectomy skin flaps. ADM was sutured to the inframammary fold at the chest wall, the fixed sized anatomical implant was inserted and the $\mathrm{ADM}$ was sutured to the pectoralis major muscle. Two suction drains were placed, draining the submuscular and subcutaneous pockets.

The surgical technique for two-stage immediate BR using temporary expander implant and later exchange to fixed size silicone implant:

A submuscular pocket was created by elevating the pectoralis major muscle, the serratus anterior muscle or its fascia and the anterior rectus sheath. A sizer was used to estimate the expansion volume and the expander was placed. Suction drains were placed, draining the submuscular and subcutaneous pockets. The first expansion was performed two weeks postoperatively and after that at weekly intervals, should no complications arise. Three to six months after the final expansion volume had been achieved the expander was changed to a fixed sized implant. The submuscular pocket was opened, the expander removed and the necessary 
adjustments of the pocket were performed. A sizer was used to determine implant size and the final size silicone implant was placed. One suction drain was used when deemed necessary. All patients received one prophylactic dose of antibiotic (Cefuroxim or Dicloxacillin) preoperatively and the one-stage group continued Cefuroxim for three days postoperatively. Drains were removed when output was less than $20 \mathrm{~mL}$ for two consecutive days.

\section{Outcomes}

Primary endpoint of the study was postoperative complications reported per breast. Explantation was defined as either loss of implant, and thereby failure of the initial implant-based BR, or as implant exchange defined as removal of the device implanted at the initial surgery and exchange to an expander. Major complications included hematoma requiring surgical intervention (within 3 days), infection requiring explantation (within three months) and mastectomy flap necrosis requiring flap revision (within four weeks). Minor complications included cellulitis/wound infection requiring treatment with antibiotics and seroma requiring intervention (within three months). For the twostage group data regarding complications following the two operations were summated in the table but described in details in the result section.

Secondary endpoints were aesthetic correction procedures, patient and investigator assessed satisfaction with the aesthetic result of the BR, symmetry and capsular contracture. All outcomes were provided for two year follow-up data. Aesthetic correction procedures included all surgical procedures conducted in general anesthesia with the aim of achieving a better aesthetic outcome for the patient. Thus, surgery due to postoperative complications was not included in this analysis. In case of unilateral BR contralateral symmetrisation procedures was also considered an aesthetic correction. Patients completed a study specific questionnaire previously used in our department $(8,9)$ as no validated Danish instrument evaluating patient satisfaction with the aesthetic result after BR was available. The internal consistency of the aesthetic satisfaction scale was found excellent (Cronbach's alpha $=0.96$ ). The questionnaire consisted of items evaluated per breast ("satisfaction with appearance of the reconstructed breast with clothes", "satisfaction with appearance of the reconstructed breast without clothes" and "overall satisfaction with the BR") and items evaluated at patient level ("satisfaction with symmetry regarding breast size" and "satisfaction with symmetry regarding breast shape"). All items were answered on a seven-point Likert scale ranging from very dissatisfied (score 1) to very satisfied (score 7). Score evaluating satisfaction with appearance of the reconstructed breast with/without clothes and scores evaluating symmetry were summated into a total score ranging from $2-14$ with higher scores representing greater aesthetic satisfaction. The investigator assessed the aesthetic outcome in regard to "overall aesthetic result of BR with clothes" and "overall aesthetic result of BR without clothes" (reported per breast) and "symmetry regarding breast size" and "symmetry regarding breast shape" (reported per patient). Assessment was scored into four categories ('excellent' score 4, 'good' score 3, 'fair' score 2, and 'poor' score 1). This was summated into a total score range $2-8$ with higher scores representing greater aesthetic satisfaction. An objective measurement of symmetry was obtained by investigator measuring suprasternal notch to nipple (SSN:N) distance and nipple to inframammary fold (N:IMF) distance using a tape measure and recorded with an accuracy of 0.5 centimetres (cm). Measurements were obtained with the patient in a standardized standing position with the arms along the side of the body. Symmetry was described as difference in $\mathrm{cm}$ between measurements of right and left side. Lower score represented a better symmetry between breasts. Furthermore, investigator assessed the degree of capsular contracture according to the Spear-Baker classification for implant based BRs (10).

\section{Bias}

The funders (financial or providing ADM) did not participate in study design, data collection, data analysis or interpretation or writing of the manuscript.

\section{Study size}

Study size was determined upon power calculation on the primary endpoint "reduction in surgery time" used for a publication in preparation. Originally 20 patients were planned in each group, but late secondary review of patients revealed, that one patient had been wrongly excluded from the one-stage group due to conversion to expander-based BR because of vulnerable mastectomy flaps and furthermore, three patients had been missed due to removal of implant before inclusion started in the twostage group. Allocating these patients to their correct study group resulted in 21 patients in the one-stage group and 23 
Table 1 Baseline demographics and clinical characteristics

\begin{tabular}{|c|c|c|}
\hline Characteristics & $\begin{array}{c}\text { One-stage, } \\
n=21\end{array}$ & $\begin{array}{c}\text { Two-stage, } \\
n=23\end{array}$ \\
\hline Age (years), mean (SD) & $48.3(10.7)$ & $42.7(9.9)$ \\
\hline $\mathrm{BMI}\left(\mathrm{kg} / \mathrm{m}^{2}\right)$, mean $(\mathrm{SD})^{\dagger}$ & $23.1(2.8)$ & $24.7(3.8)$ \\
\hline Comorbidity $^{\dagger}$ & 7 & 3 \\
\hline \multicolumn{3}{|l|}{ Smoking } \\
\hline Never smoker & 13 & 13 \\
\hline Former smoker & 6 & 10 \\
\hline Smoker & 2 & 0 \\
\hline \multicolumn{3}{|l|}{ Laterality of procedure } \\
\hline Bilateral & 11 & 6 \\
\hline Unilateral & 10 & 17 \\
\hline \multicolumn{3}{|c|}{ Adjuvant therapy after surgery ${ }^{\dagger}$} \\
\hline Endocrine treatment & 5 & 1 \\
\hline None & 15 & 19 \\
\hline \multicolumn{3}{|l|}{ Axillary surgery ${ }^{\dagger}$} \\
\hline None & 13 & 13 \\
\hline Sentinel node biopsy & 7 & 5 \\
\hline Axillary dissection ${ }^{\ddagger}$ & 0 & 2 \\
\hline \multicolumn{3}{|l|}{ Indication for mastectomy ${ }^{\dagger}$} \\
\hline Cancer & 3 & 0 \\
\hline DCIS & 4 & 6 \\
\hline Prophylactic & 13 & 14 \\
\hline
\end{tabular}

${ }^{\dagger}$, missing values one-stage group $n=1$, two-stage group $n=3 ;{ }^{\ddagger}$, two patients in the two-stage group were diagnosed with DCIS but underwent axillary dissection due to micrometastasis in sentinel nodes. BMI, body mass index; DCIS, ductal carcinoma in situ.

patients in the two-stage group.

\section{Statistical analysis}

Descriptive statistics were applied for patients' demographics giving mean and standard deviation for continuous variables. Categorical variables were compared between study arms using Fisher's exact test while continuous variables were compared by a $t$-test. For the continuous outcome the arms were compared using $t$-test for two means or were analyzed using a simple linear regression model. A multiple linear regression was used to adjust for BMI etc. Mixed regression model was used wherever it was necessary to adjust for the repeated measurements at the patient level (observations at the breast level), in which case Kenward-Roger approximation as used to adjust for the degrees of freedom due to small sample size. The dichotomous outcome was analyzed using a generalized linear model with identitylink function and in case of repeated measurements, the IDs were used as clusters. The outcome "number of aesthetic corrections" were analyzed using a Poisson regression model. Based on clinical experience it was decided to adjust for BMI and smoking in the analysis of postoperative complications. Some of the patients were dropped out after explantation (missing not randomly) and one patient was not willing to continue in the study (missing randomly). For the simplicity, all were treated missing by random and particular instances with missing values were omitted from analysis. The details of the missingness is mentioned in the results section and in relevant tables. The significance level was set to 0.05 . Statistical analyses were performed using STATA ${ }^{\circledR}$ software IC16 (Stata Corporation, College Station, TX).

\section{Results}

21 patients ( 32 breasts) were included in the one-stage group and 23 patients ( 29 breasts) were included in the two-stage group. 15 patients ( 21 breasts) in the onestage group and 19 patients ( 22 breasts) in the two-stage group completed 24 months follow-up (Figure 1). The two treatment groups did not differ significantly regarding demographics and clinical characteristics as summarized in Table 1. Three patients (three breasts) in the two-stage group and two patients (two breasts) in the one-stage group underwent prior lumpectomy. Only one patient in the one-stage group (bilateral BR) underwent nipplesparing mastectomy. Two patients in the one-stage group turned out to have smoked less than four weeks before surgery and analysis of the primary endpoint postoperative complications were adjusted for smoking. Mastectomy weight did not differ between the two groups, but there was a significant difference in final implant size (Table 2). A breast reconstructed with the two-stage technique received a final implant approximately $100 \mathrm{~mL}$ larger than a breast reconstructed with the direct to implant technique. Patients in the two-stage group underwent expansion of the expander on average 6-7 times with approximately $50 \mathrm{~mL}$ each time and the median time from insertion of expander to exchange to final size implant was 231 days. 
Table 2 Breast reconstruction descriptive data, reported per reconstructed breast

\begin{tabular}{lcc}
\hline & One-stage, breast $\mathrm{n}=32$ & Two-stage, breast $\mathrm{n}=29$ \\
\hline Mastectomy weight $(\mathrm{g})$, mean (SD) & $368(113)$ & $366(127)$ \\
Final implant size (mL), mean (SD) & $324(66)$ & $440(138)$ \\
Expander data, mean (SD) & & $95(74)$ \\
Start volume, $\mathrm{mL}$ & $6.6(1.9)$ \\
Number of expansions & $415(118)$ \\
Final expansion volume, $\mathrm{mL}$ & $231(91)$ \\
Time from expander to final implant (days), median (IQR) & & $2001^{\dagger *}$ \\
\hline
\end{tabular}

${ }^{\dagger}$, simple linear regression model; ", denotes statistical significance. IQR, interquartile range; NS, not significant.

Table 3 Postoperative complications, reported per reconstructed breast

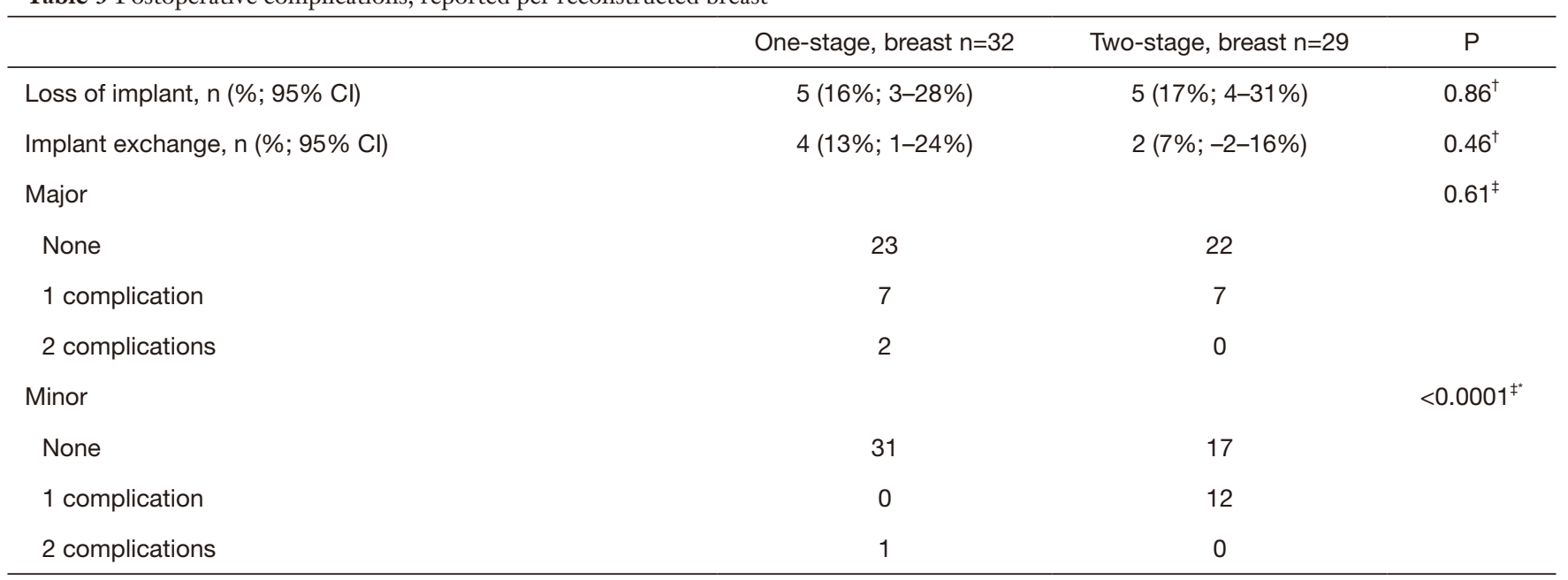

${ }^{\dagger}$, test of two proportions; ${ }^{\ddagger}$, Fisher's exact test; ${ }^{*}$, denotes statistical significance.

The risk of implant loss was equal between groups with $16 \%$ in the one-stage group compared to $17 \%$ in the twostage group (Table 3). For the two-stage group four out of five implant loss took place after exchange of expander to final size implant. The risk of implant exchange was $13 \%$ in the one-stage group compared to $7 \%$ in the two-stage group. One patient (unilateral BR) in the one-stage group was converted from direct-to-implant BR to two-stage BR after re-excision of skin due to non-radical margins and thus not because of reconstructive complications and one patient in the two-stage group (bilateral BR) underwent implant exchange to smaller implants due to discomfort. Major complications, resulting in surgery, were equally frequent in the two treatment groups. In the direct-to-implant group the risk of at least one major complication were $28 \%$ (95\% CI: $10-46 \%$ ) compared to $24 \%$ (95\% CI: $7-42 \%$ ) in the two-stage group. Major complications were equally distributed after first (two partial flap necrosis and one infection) and second operation (four infections) in the twostage group. The risk of at least one minor complication was significantly lower in the one-stage group 3\% (95\% CI: $-3-9 \%$ ) compared to the two-stage group $41 \%$ (95\% CI: $21-61 \%)$. The majority of minor complications in the twostage group were observed after insertion of the expander (five cellulitis and three seroma) and not after exchange of expander to final size implant (three cellulitis and one seroma). Adjustments for smoking and BMI did not change the results regarding complications.

There were no difference between number of aesthetic corrections between the one-stage group; mean 1.8 (95\% CI: $1.2-2.5)$ and the two-stage group; mean 1.5 (95\% CI: $0.9-2, \mathrm{P}=0.44)$. Most patients underwent at least one 
Table 4 Assessment of aesthetic result and capsular contracture, reported at breast level

\begin{tabular}{|c|c|c|c|}
\hline & One-stage, breast $n=21$ & Two-stage, breast $n=22$ & $\mathrm{P}$ \\
\hline $\begin{array}{l}\text { Investigator: assessment of overall aesthetic result of BR with/without } \\
\text { clothes (range 2-8), Mean (Cl) }\end{array}$ & $7.8(7.5-8)$ & $7.7(7.5-7.9)$ & $\mathrm{NS}^{\ddagger}$ \\
\hline Baker grade III-IV & 0 & 0 & \\
\hline
\end{tabular}

NS, not significant. ${ }^{\dagger}$, mixed regression model; ${ }^{\ddagger}$, linear regression model.

Table 5 Assessment of breast symmetry, reported at patient level

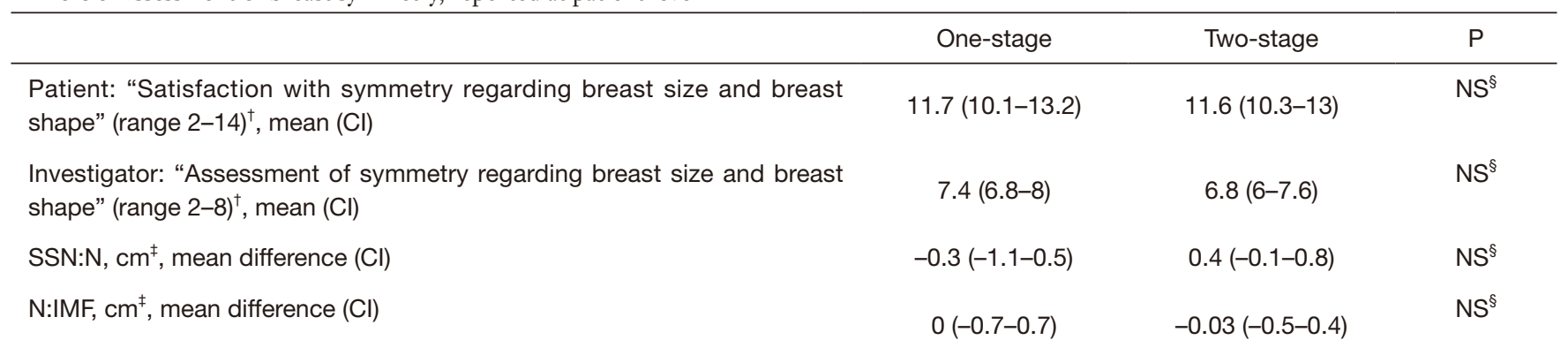

SSN:N, suprasternal notch to nipple distance; N:IMF, nipple to inframammary fold distance. ${ }^{\dagger}$, one-stage group $\mathrm{n}=15$, two stage group $\mathrm{n}=19$; ${ }^{\ddagger}$, one-stage group $\mathrm{n}=12$, two stage group $\mathrm{n}=17 ;{ }^{\S}, t$-test.

correction to get an acceptable aesthetic result and often more operations were needed. Aesthetic corrections were mostly autologous fat transplantation and symmetrization procedures. Only patients not explanted and thereby with the opportunity of receiving aesthetic corrections were included in the analysis.

Patients' satisfaction with appearance of the reconstructed breasts and the overall satisfaction with the BRs was generally very high within the range in both treatment groups (Table 4). Investigator assessment of the overall aesthetic result aligned well with the patient's assessment and was also very high in both treatment groups and there was no significant difference between the two groups. Due to lost to follow-up because of explantation analysis were performed at 21 breasts in the one-stage group and 22 breasts in the two-stage group. Assessment of symmetry was reported at patient level as symmetry requires two sides to compare (Table 5). Due to lost to follow-up because of explantation, analysis of patient and investigator assessed symmetry was performed at 15 patients in the one-stage group and 19 patients in the two-stage group. Both patients and investigator rated the symmetry very high within the range in both treatment groups without any significant difference. Measurements of SSN:N and N:IMF were only obtained if the nipple was reconstructed at the two year follow-up visit. Therefore, analysis was performed with 12 patients in the one-stage group and 17 patients in the twostage group. In both treatment groups there was less than 0.5 $\mathrm{cm}$ mean difference between left and right side in SSN:N and N:IMF measurements confirming to a very large degree symmetrical breasts (Table 5 and Figure 2). A total of $67 \%$ $(n=8)$ of patients in the one-stage group had a difference between sides at $0.5 \mathrm{~cm}$ or less in both SSN:N and N:IMF measurements. Whereas $53 \%(\mathrm{n}=9)$ in the two-stage group had a difference between sides at $0.5 \mathrm{~cm}$ or less in SSN:N measurement and 59\% $(\mathrm{n}=10)$ in N:IMF measurement. There was no significant difference between treatment groups. Significant capsular contracture, defined as Baker grade 3 or higher, was not observed in any of the treatment groups within 2 years of follow-up. 

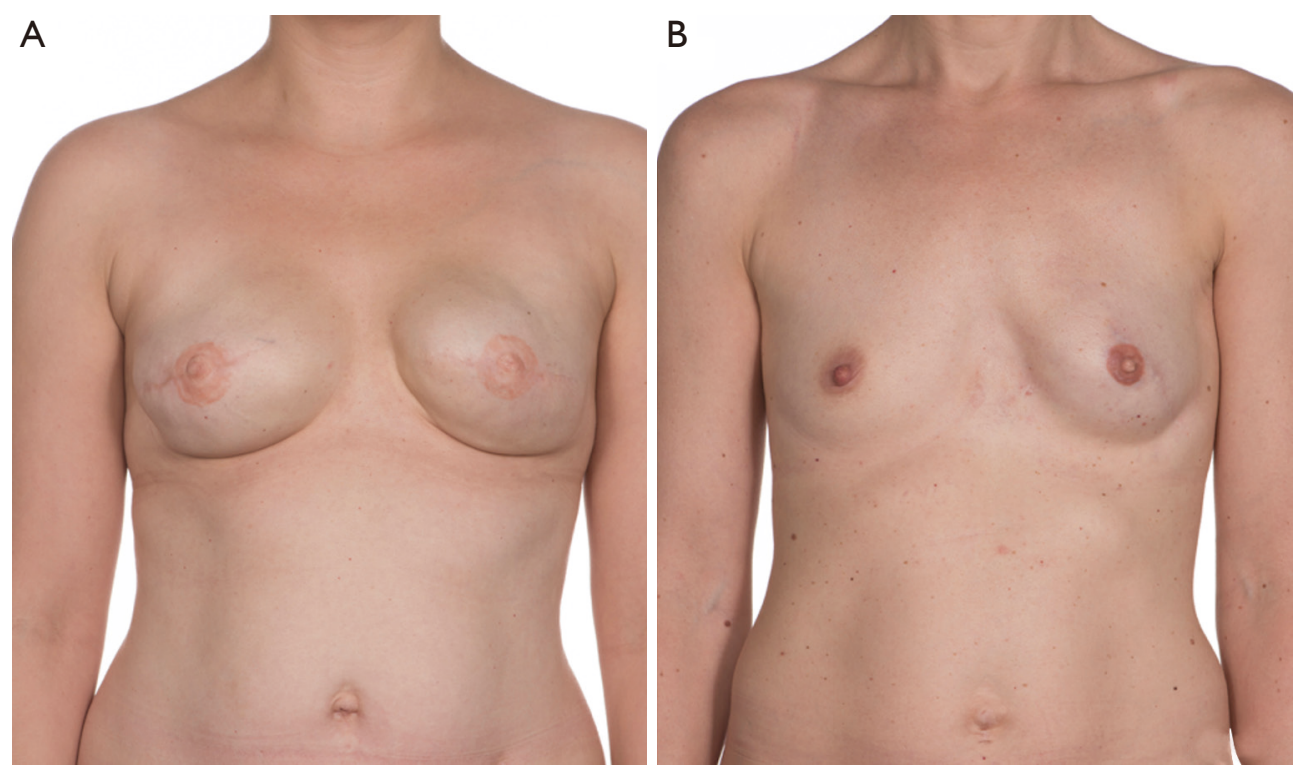

Figure 2 Two years after breast reconstruction. (A) Bilateral one-stage breast reconstruction. (B) Unilateral two-stage breast reconstruction (left).

\section{Discussion}

The present study was designed to compare two surgical techniques for implant-based BR regarding postoperative complications, aesthetic correction procedures and aesthetic outcome. Overall, no major differences in outcome was found.

Most publications do not distinguish between loss of implant and implant exchange, as we did in the present study, and comparisons can be difficult. In our view, it makes sense to separate the two outcomes as the consequences are very different. Loss of implant is considered the most severe complication, as the BR has then failed. The present study revealed no significant difference in loss of implant between the one-stage and two-stage group (16\% and 17\% respectively). But the risks was higher than observed by e.g., Potter et al. in their prospective cohort study where a risk of $8 \%$ implant loss was observed 3 months after immediate implant based BR with the use of ADM, where the majority at $86 \%$ were reconstructed as a one-stage procedure (11). In contrast Dikmans et al. revealed similar total explantation risks in their one-stage group (24\%), but a very limited risk of explantation in the two-stage group at $4 \%$ (12). Implant exchange may result in a prolonged course of BR, but not in failure of the BR. In the present study, the risk of implant exchange was not significantly different between groups.

The risk of major complications as hematoma, infection and partial flap necrosis resulting in additional surgery was equal in the two treatment groups. The risk in the one-stage group is consistent with the risk of severe adverse events for the one-stage group at $29 \%$ observed by Dikmans et al. (12) and of early revision rate at $31 \%$ observed by Gdalevitch et al. (13), but higher than observed by Potter et al. in their prospective cohort study (11). Dikmans et al. found no difference between groups regarding mild to moderate adverse events (12). This result is contradictory to what was revealed in the present study, where the risk of at least one minor complication was significantly higher in the two-stage group than in the one-stage group ( $41 \%$ $v s .3 \%)$. The number of minor complications were higher after insertion of the expander than after insertion of the final size silicone implant. It is assumed that the risk for minor complications is associated with the minimal invasive procedure at each expansion. Furthermore, the two-stage group did not receive prophylactic antibiotic treatment for three days postoperatively as the one-stage group did. This could potentially affect the risk of infectious complications. A meta-analysis including studies comparing $\mathrm{BR}$ with and without the use of $\mathrm{ADM}$ revealed, that when ADM initially was used, the risk of seroma, infection and mastectomy flap necrosis increased and the risk of capsular contracture and implant malposition decreased. This did not result in a difference in explantation risk and total complications between the groups (14). There is, however, 
in the literature a range of reported total complication rates after BR with the use of ADM with the lowest reported $3,9 \%$ (15) and 8.6\% (16). An explanation for the major and minor complication risks in the two-stage group of this study may be, that this group underwent two surgeries and multiple expansions, all of which present a risk of developing complications. Furthermore, the final implant size in the two-stage group were in average $100 \mathrm{~mL}$ larger than in the one-stage group and this might also contribute to the high rate of implant loss and complications in this treatment group. Literature suggest that increasing breast size increases the risk of complications $(13,17)$. In this study the two treatment groups had a mastectomy weight of approximately $370 \mathrm{~g}$ and this factor is not considered likely to influence the result.

Patient satisfaction with the result is crucial. Patients often undergo several surgical procedures and the end result should be worth the effort. Surgical corrections to optimize the aesthetic result of BR is most often needed. In this study most patients underwent at least one surgical procedure consisting of mostly autologous fat grafting, but also skin reduction and contralateral reduction to achieve symmetry. Autologous fat grafting has been showed to improve the cosmetic outcome after implant based BR and furthermore alleviate pain $(18,19)$ and is considered to be a low risk intervention. In this study patients reported a very high overall satisfaction with the BR regardless of which surgical approach was used. Likewise, both patients and investigator were very satisfied with the overall aesthetic result in both treatment groups. This result is contradictory to other studies where satisfaction with the aesthetic result of BR were higher when performed with the use of $\operatorname{ADM}(20,21)$. An explanation for the very high satisfaction might be, that the reconstructive surgeons provided the patients with both orally and written detailed information prior to surgery to obtain a common understanding of the reconstructive course and expected outcome. Literature suggests this to be one of the main factors for a high satisfaction with the result of BR. Breast symmetry does also play an important role for satisfaction, but is not found to be a major determinant of outcome (22). Breast asymmetry is a common phenomenon and the proportions of patients in this study that obtained an acceptable symmetri of $0.5 \mathrm{~cm}$ or less difference in SSN:N measurement was in accordance with a normal population opting for BR (23). Absolut symmetry is not the ultimate goal and patients were informed of this prior to surgery in an attempt to manage patients' expectations.

Breasts reconstructed with the two-stage method received a significantly larger final implant than breasts reconstructed with the one-stage procedure. An explanation for this finding was that patients undergoing bilateral $\mathrm{BR}$ received larger implants due to patient request. This difference between groups was not reflected in assessment of satisfaction with the BR. It is suggested, that in general it is of less importance whether the reconstructed breast is larger or smaller than the former natural breast as long as symmetry is achieved. The gradual expansion of skin and muscle tissue in the two-stage group ultimately gives the possibility to insert larger implants with the tradeoff of more outpatient visits and an additional surgery under general anesthesia.

Capsular contracture may affect the aesthetic outcome and cause pain or discomfort. The phenomenon is observed to be a progressive condition but in $\mathrm{BR}$ with $\mathrm{ADM}$, literature suggests that capsular contraction appears within a period of two years after BR (24). Furthermore, a comparison between immediate two-stage BR with and without ADM concluded, that the use of ADM was associated with less capsular contracture. Risk with/without ADM was 3.8\% and $19.4 \%$ respectively (OR 0.18 ) (20). Research into the mechanisms of how ADM influences the development of capsular contracture is limited. In a recent study more myofibroblast and neovascularization were observed in the capsule of breasts reconstructed with biological mesh than in breasts reconstructed with synthetic mesh. Furthermore, collagen fibers seemed to be aligned in an irregular pattern with both parallel and vertical fibers (25). However, this difference in formation of capsular tissue was not reflected in the frequency of capsular contracture maybe due to a follow-up time of less than two years. In this study no capsular contraction grade III or IV was observed within two years follow-up in any of the treatment groups.

A limitation for this study is the small study population. Sample size was determined upon the endpoint surgery time which is not part of this publication. Consequently, large confidence intervals was observed and missing values could not be handled in any other way, e.g., imputation. Furthermore, no validated questionnaire as e.g., Breast-Q was used for measuring satisfaction with the result as this questionnaire was not available in a validated danish version at the time of study start.

It is a strength that all BR was performed by the same three experienced plastic surgeons and four breast oncology surgeons during the entire study period. They did not participate in the evaluation of the aesthetic result and there was only one independent investigator that did not 
participate in the surgical procedures. Introduction of a new surgical technique result in a learning curve as experience is achieved. It has previously been suggested that the learning curve may have influence on the complication rate after BR with $\mathrm{ADM}$ (26). This factor might be of importance in the present study. Two years follow-up time is thought to be an appropriate time for assessment of secondary endpoints.

An important prerequisites of a successful immediate $\mathrm{BR}$ is viable mastectomy skin flaps. This emphasizes that the collaboration between plastic- and breast surgeons is of pivotal importance. Imaging techniques, for instance laserassisted indocyanine green angiography, can also potentially decrease complication rates. It is a useful technique to identify mastectomy skinflap areas with insufficient perfusion even though our institution did not find a beneficial effect on necrosis rates after implementing this technique (27).

Since this study was initiated, prepectoral breast reconstructive procedures $\mathrm{ADM}$ has been developed. It is a less invasive procedure and the advantages includes reduced pain and less animation deformities (28). The prepectoral approach may result in a secondary procedure with subcutaneous fat grafting to achieve a satisfying aesthetic result and furthermore, the expense of ADM may also be a factor to consider (29). Nipple-sparing mastectomy further enhances the cosmetic outcome and satisfaction with the BR (30).

Further research should be undertaken to investigate the cost effectiveness of the two treatment methods described in this study. ADM is expensive and the costs should be offset by fewer complications, better outcome or a lower burden on the health care system and the patient.

\section{Conclusions}

The present study design sets limitations for drawing wide conclusions. Since this study was initiated a randomized study has concluded, that that use of one-stage BR with ADM should be considered carefully due to more early postoperative complications than after two-stage BR (12). The present study did not reveal any significant differences between the two breast reconstructive techniques besides a higher risk of minor complications in the two-stage group, that did, however, not lead to a higher risk of implant loss. With equally high satisfaction with the aesthetic result and no significant difference in number of aesthetic corrections between the two groups, we find, based on this study, that the one-stage approach using ADM may be feasible and allows the patient to achieve an implant-based BR with a minimum of surgeries and outpatient visits.

\section{Acknowledgments}

The authors wish to express their gratitude to the participating patients.

Funding: This work was supported by the Danish Cancer Society [R130-A8304-15-S38]; the Korning foundation; Foundation of the Kjaersgaard Family, Sunds; King Christian X foundation; Foundation of Architect Holger Hjortenberg and LifeCell Corporation (Branchburg, NJ, USA). Furthermore, LifeCell Corporation LifeCell Corporation (Branchburg, NJ, USA) provided acellular dermal matrix (Strattice ${ }^{\mathrm{TM}}$ ) for the study. None of the financial contributors had any involvement in the study design, data analysis or interpretation of the results.

\section{Footnote}

Reporting Checklist: The authors have completed the STROBE reporting checklist. Available at http://dx.doi. org/10.21037/gs-20-581

Data Sharing Statement: Available at http://dx.doi. org/10.21037/gs-20-581

Peer Review File: Available at http://dx.doi.org/10.21037/gs20-581

Conflicts of Interest: All authors have completed the ICMJE uniform disclosure form (available at http:// dx.doi.org/10.21037/gs-20-581). MB reports grants from The Danish Cancer Society, grants from The Korning foundation, grants from Foundation of the Kjaersgaard Family, Sunds, grants from King Christian X foundation, grants from Foundation of Architect Holger Hjortenberg, grants and non-financial support from LifeCell Corporation (Branchburg, NJ, USA), during the conduct of the study. The other authors have no conflicts of interest to declare.

Ethical Statement: The authors are accountable for all aspects of the work in ensuring that questions related to the accuracy or integrity of any part of the work are appropriately investigated and resolved. The trial was conducted in accordance with the Declaration of Helsinki (as revised in 2013) and the Harmonized Tripartite Guideline for Good Clinical Practice from the International 
Conference on Harmonization. This study was reviewed and approved by the Ethics Committee of the Central Region of Denmark (1-10-72-572-12) and informed consent was taken from all the patients.

Open Access Statement: This is an Open Access article distributed in accordance with the Creative Commons Attribution-NonCommercial-NoDerivs 4.0 International License (CC BY-NC-ND 4.0), which permits the noncommercial replication and distribution of the article with the strict proviso that no changes or edits are made and the original work is properly cited (including links to both the formal publication through the relevant DOI and the license). See: https://creativecommons.org/licenses/by-nc-nd/4.0/.

\section{References}

1. Breuing KH, Warren SM. Immediate bilateral breast reconstruction with implants and inferolateral AlloDerm slings. Ann Plast Surg 2005;55:232-9.

2. Namnoum JD. Expander/implant reconstruction with AlloDerm: recent experience. Plast Reconstr Surg 2009;124:387-94.

3. Breuing KH, Colwell AS. Inferolateral AlloDerm hammock for implant coverage in breast reconstruction. Ann Plast Surg 2007;59:250-5.

4. Cordeiro PG. Breast reconstruction after surgery for breast cancer. N Engl J Med 2008;359:1590-601.

5. Hallberg H, Rafnsdottir S, Selvaggi G, et al. Benefits and risks with acellular dermal matrix (ADM) and mesh support in immediate breast reconstruction: a systematic review and meta-analysis. J Plast Surg Hand Surg 2018;52:130-47.

6. Colakoglu S, Khansa I, Curtis MS, et al. Impact of complications on patient satisfaction in breast reconstruction. Plast Reconstr Surg 2011;127:1428-36.

7. Toyoda Y, Fu RH, Li L, et al. Smoking as an Independent Risk Factor for Postoperative Complications in Plastic Surgical Procedures: A Propensity Score-Matched Analysis of 36,454 Patients from the NSQIP Database from 2005 to 2014. Plast Reconstr Surg 2018;141:226-36.

8. Juhl AA, Christensen S, Zachariae R, et al. Unilateral breast reconstruction after mastectomy - patient satisfaction, aesthetic outcome and quality of life. Acta Oncol 2017;56:225-31.

9. Christensen BO, Overgaard J, Kettner LO, et al. Longterm evaluation of postmastectomy breast reconstruction. Acta Oncol 2011;50:1053-61.
10. Spear SL, Baker JL Jr. Classification of capsular contracture after prosthetic breast reconstruction. Plast Reconstr Surg 1995;96:1119-23; discussion 1124.

11. Potter S, Conroy EJ, Cutress RI, et al. Short-term safety outcomes of mastectomy and immediate implant-based breast reconstruction with and without mesh (iBRA): a multicentre, prospective cohort study. Lancet Oncol 2019;20:254-66.

12. Dikmans RE, Negenborn VL, Bouman MB, et al. Twostage implant-based breast reconstruction compared with immediate one-stage implant-based breast reconstruction augmented with an acellular dermal matrix: an open-label, phase 4, multicentre, randomised, controlled trial. Lancet Oncol 2017;18:251-8.

13. Gdalevitch P, Ho A, Genoway K, et al. Direct-to-implant single-stage immediate breast reconstruction with acellular dermal matrix: predictors of failure. Plast Reconstr Surg 2014;133:738e-47e.

14. Lee KT, Mun GH. Updated Evidence of Acellular Dermal Matrix Use for Implant-Based Breast Reconstruction: A Meta-analysis. Ann Surg Oncol 2016;23:600-10.

15. Salzberg CA, Ashikari AY, Koch RM, et al. An 8-year experience of direct-to-implant immediate breast reconstruction using human acellular dermal matrix (AlloDerm). Plast Reconstr Surg 2011;127:514-24.

16. Hunsicker LM, Ashikari AY, Berry C, et al. Short-Term Complications Associated With Acellular Dermal MatrixAssisted Direct-to-Implant Breast Reconstruction. Ann Plast Surg 2017;78:35-40.

17. Negenborn VL, Dikmans REG, Bouman MB, et al. Predictors of complications after direct-to-implant breast reconstruction with an acellular dermal matrix from a multicentre randomized clinical trial. Br J Surg 2018;105:1305-12.

18. Cogliandro A, Barone M, Tenna S, et al. The Role of Lipofilling After Breast Reconstruction: Evaluation of Outcomes and Patient Satisfaction with BREAST-Q. Aesthetic Plast Surg 2017;41:1325-31.

19. Juhl AA, Karlsson P, Damsgaard TE. Fat grafting for alleviating persistent pain after breast cancer treatment: A randomized controlled trial. J Plast Reconstr Aesthet Surg 2016;69:1192-202.

20. Vardanian AJ, Clayton JL, Roostaeian J, et al. Comparison of implant-based immediate breast reconstruction with and without acellular dermal matrix. Plast Reconstr Surg 2011;128:403e-10e.

21. Forsberg CG, Kelly DA, Wood BC, et al. Aesthetic outcomes of acellular dermal matrix in tissue expander/ 
implant-based breast reconstruction. Ann Plast Surg 2014;72:S116-20.

22. Yip JM, Watson DI, Tiggemann M, et al. Determinants of breast reconstruction outcome: How important is volume symmetry? J Plast Reconstr Aesthet Surg 2015;68:679-85.

23. Cheong AL, Liu J, Reece GP, et al. Natural Breast Symmetry in Preoperative Breast Cancer Patients. Plast Reconstr Surg Glob Open 2019;7:e2297.

24. Salzberg CA, Ashikari AY, Berry C, et al. Acellular Dermal Matrix-Assisted Direct-to-Implant Breast Reconstruction and Capsular Contracture: A 13-Year Experience. Plast Reconstr Surg 2016;138:329-37.

25. Hansson E, Burian P, Hallberg H. Comparison of inflammatory response and synovial metaplasia in immediate breast reconstruction with a synthetic and a biological mesh: a randomized controlled clinical trial. J Plast Surg Hand Surg 2020;54:131-6.

26. Lardi AM, Ho-Asjoe M, Mohanna PN, et al. Immediate

Cite this article as: Brunbjerg ME, Jensen TB, Overgaard J, Christiansen P, Damsgaard TE. Comparison of one-stage direct-to-implant with acellular dermal matrix and two-stage immediate implant-based breast reconstruction-a cohort study. Gland Surg 2021;10(1):207-218. doi: 10.21037/gs-20-581 breast reconstruction with acellular dermal matrix: factors affecting outcome. J Plast Reconstr Aesthet Surg 2014;67:1098-105.

27. Hammer-Hansen N, Juhl AA, Damsgaard TE. Laserassisted indocyanine green angiography in implant-based immediate breast reconstruction: a retrospective study. J Plast Surg Hand Surg 2018;52:158-62.

28. Sbitany H, Piper M, Lentz R. Prepectoral Breast Reconstruction: A Safe Alternative to Submuscular Prosthetic Reconstruction following Nipple-Sparing Mastectomy. Plast Reconstr Surg 2017;140:432-43.

29. Margulies IG, Salzberg CA. The use of acellular dermal matrix in breast reconstruction: evolution of techniques over 2 decades. Gland Surg 2019;8:3-10.

30. Didier F, Radice D, Gandini S, et al. Does nipple preservation in mastectomy improve satisfaction with cosmetic results, psychological adjustment, body image and sexuality? Breast Cancer Res Treat 2009;118:623-33. 